\title{
Tangles and Connectivity in Graphs
}

\author{
Martin Grohe \\ RWTH Aachen University \\ grohe@informatik.rwth-aachen.de
}

\begin{abstract}
This paper is a short introduction to the theory of tangles, both in graphs and general connectivity systems. An emphasis is put on the correspondence between tangles of order $k$ and $k$-connected components. In particular, we prove that there is a one-to-one correspondence between the triconnected components of a graph and its tangles of order 3 .
\end{abstract}

\section{Introduction}

Tangles, introduced by Robertson and Seymour in the tenth paper [21] of their graph minors series [20, have come to play an important part in structural graph theory. For example, Robertson and Seymour's structure theorem for graphs with excluded minors is phrased in terms of tangles in its general form 22. Tangles have also played a role in algorithmic structural graph theory (for example in [3, 7, 8, 11, 14).

Tangles describe highly connected regions in a graph. In a precise mathematical sense, they are "dual" to decompositions (see Theorem 6.1). Intuitively, a graph has a highly connected region described by a tangle if and only if it does not admit a decomposition along separators of low order. By decomposition I always mean a decomposition in a treelike fashion; formally, this is captured by the notions of tree decomposition or branch decomposition.

However, tangles describe regions of a graph in an indirect and elusive way. This is why we use the unusual term "region" instead of "subgraph" or "component". The idea is that a tangle describes a region by pointing to it. A bit more formally, a tangle of order $k$ assigns a "big side" to every separation of order less than $k$. The big side is where the (imaginary) region described by the tangle is supposed to be. Of course this assignment of "big sides" to the separations is subject to certain consistency and nontriviality conditions, the "tangle axioms".

To understand why this way of describing a "region" is a good idea, let us review decompositions of graphs into their $k$-connected components. It is well known that every graph can be decomposed into its connected components and into its biconnected components. The former are the (inclusionwise) maximal connected subgraphs, and the latter the maximal 2-connected subgraphs. It is also well-known that a graph can be decomposed into its triconnected components, but the situation is more complicated here. Different from what one might guess, the triconnected components are not maximal 3-connected subgraphs; in fact they are not even subgraphs, but just topological subgraphs (see Section 2 for a definition of topological subgraphs). Then what about 4-connected components?

It turns out that in general a graph does not have a reasonable decomposition into 4-connected components (neither into $k$-connected components for any $k \geq 5$ ), at least if these components are supposed to be 4-connected and some kind of subgraph. To understand the difficulty, consider the hexagonal grid in Figure 1.1. It is 3-connected, 


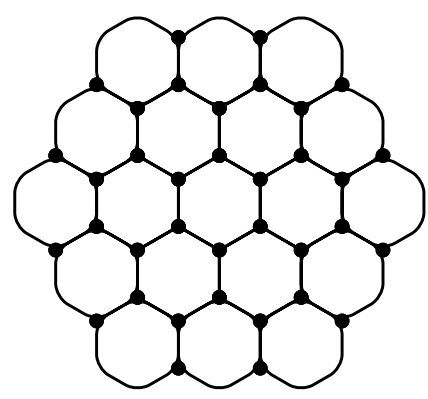

Figure 1.1. A hexagonal grid

but not 4-connected. In fact, for any two nonadjacent vertices there is a separator of order 3 separating these two vertices. Thus it is not clear what the 4-connected components of a grid could possibly be (except, of course, just the single vertices, but this would not lead to a meaningful decomposition). But maybe we need to adjust our view on connectivity: a hexagonal grid is fairly highly connected in a "global sense". All its low-order separations are very unbalanced. In particular, all separations of order 3 have just a single vertex on one side and all other vertices on the other side. This type of global connectivity is what tangles are related to. For example, there is a unique tangle of order 4 in the hexagonal grid: the big side of a separation of order 3 is obviously the side that contains all but one vertex. The "region" this tangle describes is just the grid itself. This does not sound particularly interesting, but the grid could be a subgraph of a larger graph, and then the tangle would identify it as a highly connected region within that graph. A key theorem about tangles is that every graph admits a canonical tree decomposition into its tangles of order $k$ [1, 21. This can be seen as a generalisation of the decomposition of a graph into its 3-connected components. A different, but related generalisation has been given in [2].

The theory of tangles and decompositions generalises from graphs to an abstract setting of connectivity systems. This includes nonstandard notions of connectivity on graphs, such as the "cut-rank" function, which leads to the notion of "rank width" 16, 17, and connectivity functions on other structures, for example matroids. Tangles give us an abstract notion of "k-connected components" for these connectivity systems. The canonical decomposition theorem can be generalised from graphs to this abstract setting [5, 13.

This paper is a short introduction to the basic theory of tangles, both for graphs and for general connectivity systems. We put a particular emphasis on the correspondence between tangles of order $k$ and $k$-connected components of a graph for $k \leq 3$, which gives some evidence to the claim that for all $k$, tangles of order $k$ may be viewed as a formalisation of the intuitive notion of " $k$-connected component".

The paper provides background material for my talk at LATA. The talk itself will be concerned with more recent results [6] and, in particular, computational aspects and applications of tangles [9, 10, 11].

\section{Preliminaries}

We use a standard terminology and notation (see 4 for background); let me just review a few important notions. All graphs considered in this paper are finite and simple. The vertex set and edge set of a graph $G$ are denoted by $V(G)$ and $E(G)$, respectively. The order of $G$ is $|G|:=|V(G)|$. For a set $W \subseteq V(G)$, we denote the induced subgraph of 
$G$ with vertex set $W$ by $G[W]$ and the induced subgraph with vertex set $V(G) \backslash W$ by $G \backslash W$. The (open) neighbourhood of a vertex $v$ in $G$ is denoted by $N^{G}(v)$, or just $N(v)$ if $G$ is clear from the context. For a set $W \subseteq V(G)$ we let $N(W):=\left(\bigcup_{v \in W} N(v)\right) \backslash W$, and for a subgraph $H \subseteq G$ we let $N(H):=N(V(H))$. The union of two graphs $A, B$ is the graph $A \cup B$ with vertex set $V(A) \cup V(B)$ and edge set $E(A) \cup E(B)$, and the intersection $A \cap B$ is defined similarly.

A separation of $G$ is a pair $(A, B)$ of subgraphs of $G$ such that $A \cup B=G$ and $E(A) \cap E(B)=\emptyset$. The order of the separation $(A, B)$ is $\operatorname{ord}(A, B):=|V(A) \cap V(B)|$. A separation $(A, B)$ is proper if $V(A) \backslash V(B)$ and $V(B) \backslash V(A)$ are both nonempty. A graph $G$ is $k$-connected if $|G|>k$ and $G$ has no proper $(k-1)$-separation.

A subdivision of $G$ is a graph obtained from $G$ by subdividing some (or all) of the edges, that is, replacing them by paths of length at least 2. A graph $H$ is a topological subgraph of $G$ if a subdivision of $H$ is a subgraph of $G$.

\section{Tangles in a Graph}

In this section we introduce tangles of graphs, give a few examples, and review a few basic facts about tangles, all well-known and at least implicitly from Robertson and Seymour's fundamental paper on tangles [21] (except Theorem 3.7 which is due to Reed [19]).

Let $G$ be a graph. A $G$-tangle of order $k$ is a family $\mathcal{T}$ of separations of $G$ satisfying the following conditions.

(GT.0) The order of all separations $(A, B) \in \mathcal{T}$ is less than $k$.

(GT.1) For all separations $(A, B)$ of $G$ of order less than $k$, either $(A, B) \in \mathcal{T}$ or $(B, A) \in \mathcal{T}$.

(GT.2) If $\left(A_{1}, B_{1}\right),\left(A_{2}, B_{2}\right),\left(A_{3}, B_{3}\right) \in \mathcal{T}$ then $A_{1} \cup A_{2} \cup A_{3} \neq G$.

(GT.3) $V(A) \neq V(G)$ for all $(A, B) \in \mathcal{T}$.

Observe that (GT.1) and (GT.2) imply that for all separations $(A, B)$ of $G$ of order less than $k$, exactly one of the separations $(A, B),(B, A)$ is in $\mathcal{T}$.

We denote the order of a tangle $\mathcal{T}$ by $\operatorname{ord}(\mathcal{T})$.

Example 3.1. Let $G$ be a graph and $C \subseteq G$ a cycle. Let $\mathcal{T}$ be the set of all separations $(A, B)$ of $G$ of order 1 such that $C \subseteq B$. Then $\mathcal{T}$ is a $G$-tangle of order 2 .

To see this, note that $\mathcal{T}$ trivially satisfies (GT.0), It satisfies (GT.1), because for every separation $(A, B)$ of $G$ of order 1 , either $C \subseteq A$ or $C \subseteq B$. To see that $\mathcal{T}$ satisfies (GT.3), let $\left(A_{i}, B_{i}\right) \in \mathcal{T}$ for $i=1,2,3$. Note that it may happen that $V\left(A_{1}\right) \cup V\left(A_{2}\right) \cup$ $V\left(A_{3}\right)=V(G)$ (if $|C|=3$ ). However, no edge of $C$ can be in $E\left(A_{i}\right)$ for any $i$, because $C \subseteq B_{i}$ and $\left|A_{i} \cap B_{i}\right| \leq 1$. Hence $E\left(A_{1}\right) \cup A\left(A_{2}\right) \cup E\left(A_{3}\right) \neq E(G)$, which implies (GT.2) Finally, $\mathcal{T}$ satisfies (GT.3), because $V(C) \backslash V(A) \neq \emptyset$ for all $(A, B) \in \mathcal{T}$.

Example 3.2. Let $G$ be a graph and $X \subseteq V(G)$ a clique in $G$. Note that for all separations $(A, B)$ of $G$, either $X \subseteq V(A)$ or $X \subseteq V(B)$. For every $k \geq 1$, let $\mathcal{T}_{k}$ be the set of all separations $(A, B)$ of $G$ of order less than $k$ such that $X \subseteq V(B)$.

Then if $k<\frac{2}{3}|X|+1$, the set $\mathcal{T}_{k}$ is a $G$-tangle of order $k$. We omit the proof, which is similar to the proof in the previous example.

Instead, we prove that $\mathcal{T}_{k}$ is not necessarily a $G$-tangle if $k=\frac{2}{3}|X|+1$. To see this, let $G$ be a complete graph of order $3 n, k:=2 n+1$, and $X:=V(G)$. Suppose for contradiction that $\mathcal{T}_{k}$ is a $G$-tangle of order $k$. Partition $X$ into three sets $X_{1}, X_{2}, X_{3}$ of size $n$. For $i \neq j$, let $A_{i j}:=G\left[X_{i} \cup X_{j}\right]$ and $B_{i j}:=G$. Then $\left(A_{i j}, B_{i j}\right)$ is a separation of $G$ of order $2 n<k$. By (GT.1) and (GT.3)] we have $\left(A_{i j}, B_{i j}\right) \in \mathcal{T}_{k}$. However, $A_{12} \cup A_{13} \cup A_{23}=G$, and this contradicts (GT.2). 


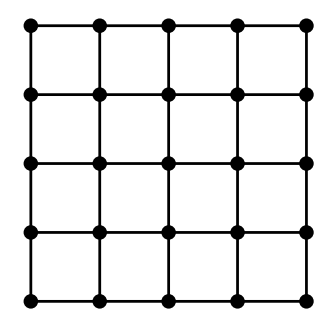

Figure 3.1. A $(5 \times 5)$-grid

Example 3.3. Let $G$ be a graph and $H \subseteq G$ a $(k \times k)$-grid (see Figure 3.1). Let $\mathcal{T}$ be the set of all separations $(A, B)$ of $G$ of order at most $k-1$ such that $B$ contains some row of the grid. Then $\mathcal{T}$ is a $G$-tangle of order $k$. (See [21] for a proof.)

The reader may wonder why in (GT.2) we take three separations, instead of two or four or seventeen. The following lemma gives (some kind of) an explanation: we want our tangles to be closed under intersection, in the weak form stated as assertion (3) of the lemma; this is why taking just two separations in (GT.2) would not be good enough. Three is just enough, and as we do not want to be unnecessarily restrictive, we do not take more than three separations.

Lemma 3.4. Let $\mathcal{T}$ be a $G$-tangle of order $k$.

(1) If $(A, B)$ is a separation of $G$ with $|V(A)|<k$ then $(A, B) \in \mathcal{T}$.

(2) If $(A, B) \in \mathcal{T}$ and $\left(A^{\prime}, B^{\prime}\right)$ is a separation of $G$ of order $<k$ such that $B^{\prime} \supseteq B$, then $\left(A^{\prime}, B^{\prime}\right) \in \mathcal{T}$.

(3) If $(A, B),\left(A^{\prime}, B^{\prime}\right) \in \mathcal{T}$ and $\operatorname{ord}\left(A \cup A^{\prime}, B \cap B^{\prime}\right)<k$ then $\left(A \cup A^{\prime}, B \cap B^{\prime}\right) \in \mathcal{T}$.

Proof. We leave the proofs of (1) and (2) to the reader. To prove (3), let $(A, B),\left(A^{\prime}, B^{\prime}\right) \in$ $\mathcal{T}$ and $\operatorname{ord}\left(A \cup A^{\prime}, B \cap B^{\prime}\right)<k$. By (GT.1), either $\left(A \cup A^{\prime}, B \cap B^{\prime}\right) \in \mathcal{T}$ or $\left(B \cup B^{\prime}, A \cap A^{\prime}\right) \in$ $\mathcal{T}$. As $A \cup A^{\prime} \cup\left(B \cup B^{\prime}\right)=G$, by (GT.2) we cannot have $\left(B \cup B^{\prime}, A \cap A^{\prime}\right) \in \mathcal{T}$.

Corollary 3.5. Let $\mathcal{T}$ be a $G$-tangle of order $k$. Let $(A, B),\left(A^{\prime}, B^{\prime}\right) \in \mathcal{T}$. Then $\mid B \cap$ $B^{\prime} \mid \geq k$.

The following lemma will allow us, among other things, to give an alternative characterisation of tangles in terms of so-called brambles.

Lemma 3.6. Let $\mathcal{T}$ be a $G$-tangle of order $k$. Then for every set $S \subseteq V(G)$ of cardinality $|S|<k$ there is a unique connected component $C(\mathcal{T}, S)$ of $G \backslash S$ such that for all separations $(A, B)$ of $G$ with $V(A) \cap V(B) \subseteq S$ we have $(A, B) \in \mathcal{T} \Longleftrightarrow C(\mathcal{T}, S) \subseteq B$.

Proof. Let $C_{1}, \ldots, C_{m}$ be the set of all connected components of $G \backslash S$. For every $I \subseteq[m]$, let $C_{I}:=\bigcup_{i \in I} C_{i}$. We define a separation $\left(A_{I}, B_{I}\right)$ of $G$ as follows. $B_{I}$ is the graph with vertex set $S \cup V\left(C_{I}\right)$ and all edges that have at least one endvertex in $V\left(C_{I}\right)$, and $A_{I}$ is the graph with vertex set $S \cup V\left(C_{[m] \backslash I}\right)$ and edge set $E(G) \backslash E\left(B_{I}\right)$. Note that $V\left(A_{I}\right) \cap V\left(B_{I}\right)=S$ and thus ord $\left(A_{I}, B_{I}\right)<k$. Thus for all $I$, either $\left(A_{I}, B_{I}\right) \in \mathcal{T}$ or $\left(B_{I}, A_{I}\right) \in \mathcal{T}$. It follows from Lemma 3.4(1) and (GT.2) that $\left(B_{I}, A_{I}\right) \in \mathcal{T}$ implies $\left(A_{[m] \backslash I}, B_{[m] \backslash I}\right) \in \mathcal{T}$, because $(G[S], G) \in \mathcal{T}$ and $B_{I} \cup B_{[m] \backslash I} \cup G[S]=G$. Furthermore, it follows from Lemma $3.4(3)$ that $\left(A_{I}, B_{I}\right),\left(A_{J}, B_{J}\right) \in \mathcal{T}$ implies $\left(A_{I \cap J}, B_{I \cap J}\right) \in \mathcal{T}$. By (GT.3) we have $\left(A_{[m]}, B_{[m]}\right) \in \mathcal{T}$ and $\left(A_{\emptyset}, B_{\emptyset}\right) \notin \mathcal{T}$. 
Let $I \subseteq[m]$ be of minimum cardinality such that $\left(A_{I}, B_{I}\right) \in \mathcal{T}$. Since $\left(A_{I}, B_{I}\right),\left(A_{J}, B_{J}\right) \in$ $\mathcal{T}$ implies $\left(A_{I \cap J}, B_{I \cap J}\right) \in \mathcal{T}$, the minimum set $I$ is unique. If $|I|=1$, then we let $C(\mathcal{T}, S):=C_{i}$ for the unique element $i \in I$. Suppose for contradiction that $|I|>1$, and let $i \in I$. By the minimality of $|I|$ we have $\left(A_{\{i\}}, B_{\{i\}}\right) \notin \mathcal{T}$ and thus $\left(A_{[m] \backslash\{i\}}, B_{[m] \backslash\{i\}}\right) \in \mathcal{T}$. This implies $\left(A_{I \backslash\{i\}}, B_{I \backslash\{i\}}\right) \in \mathcal{T}$, contradicting the minimality of $|I|$.

Let $G$ be a graph. We say that subgraphs $C_{1}, \ldots, C_{m} \subseteq G$ touch if there is a vertex $v \in \bigcap_{i=1}^{m} V\left(C_{i}\right)$ or an edge $e \in E(G)$ such that each $C_{i}$ contains at least one endvertex of $e$. A family $\mathcal{C}$ of subgraphs of $G$ touches pairwise if all $C_{1}, C_{2} \in \mathcal{C}$ touch, and it touches triplewise if all $C_{1}, C_{2}, C_{3} \in \mathcal{C}$ touch. A vertex cover (or hitting set) for $\mathcal{C}$ is a set $S \subseteq V(G)$ such that $S \cap V(C) \neq \emptyset$ for all $C \in \mathcal{C}$.

Theorem 3.7 (Reed [19]). A graph $G$ has a $G$-tangle of order $k$ if and only if there is a family $\mathcal{C}$ of connected subgraphs of $G$ that touches triplewise and has no vertex cover of cardinality less than $k$.

In fact, Reed [19] defines a tangle of a graph $G$ to be a family $\mathcal{C}$ of connected subgraphs of $G$ that touches triplewise and its order to be the cardinality of a minimum vertex cover. A bramble is a family $\mathcal{C}$ of connected subgraphs of $G$ that touches pairwise. In this sense, a tangle is a special bramble.

of Theorem 3.7. For the forward direction, let $\mathcal{T}$ be a $G$-tangle of order $k$. We let

$$
\mathcal{C}:=\{C(\mathcal{T}, S) \mid S \subseteq V(G) \text { with }|S|<k\} .
$$

$\mathcal{C}$ has no vertex cover of cardinality less than $k$, because if $S \subseteq V(G)$ with $|S|<k$ then $S \cap V(C(\mathcal{T}, S))=\emptyset$. It remains to prove that $\mathcal{C}$ touches triplewise. For $i=1,2,3$, let $C_{i} \in \mathcal{C}$ and $S_{i} \subseteq V(G)$ with $\left|S_{i}\right|<k$ such that $C_{i}=C\left(\mathcal{T}, S_{i}\right)$. Let $B_{i}$ be the graph with vertex set $V\left(C_{i}\right) \cup S$ and all edges of $G$ that have at least one vertex in $V\left(C_{i}\right)$, and let $A_{i}$ be the graph with vertex set $V(G) \backslash V\left(C_{i}\right)$ and the remaining edges of $G$. Since $C\left(\mathcal{T}, S_{i}\right)=C_{i} \subseteq B_{i}$, we have $\left(A_{i}, B_{i}\right) \in \mathcal{T}$. Hence $A_{1} \cup A_{2} \cup A_{3} \neq G$ by (GT.2), and this implies that $C_{1}, C_{2}, C_{3}$ touch.

For the backward direction, let $\mathcal{C}$ be a family of connected subgraphs of $G$ that touches triplewise and has no vertex cover of cardinality less than $k$. We let $\mathcal{T}$ be the set of all separations $(A, B)$ of $G$ of order less than $k$ such that $C \subseteq B \backslash V(A)$ for some $C \in \mathcal{C}$. It is easy to verify that $\mathcal{T}$ is a $G$-tangle of order $k$.

Let $\mathcal{T}, \mathcal{T}^{\prime}$ be $\kappa$-tangles. If $\mathcal{T}^{\prime} \subseteq \mathcal{T}$, we say that $\mathcal{T}$ is an extension of $\mathcal{T}^{\prime}$. The truncation of $\mathcal{T}$ to order $k \leq \operatorname{ord}(\mathcal{T})$ is the set $\{(A, B) \in \mathcal{T} \mid \operatorname{ord}(A, B)<k\}$, which is obviously a tangle of order $k$. Observe that if $\mathcal{T}$ is an extension of $\mathcal{T}^{\prime}$, then $\operatorname{ord}\left(\mathcal{T}^{\prime}\right) \leq \operatorname{ord}(\mathcal{T})$, and $\mathcal{T}^{\prime}$ is the truncation of $\mathcal{T}$ to order $\operatorname{ord}\left(\mathcal{T}^{\prime}\right)$.

\section{Tangles and Components}

In this section, we will show that there is a one-to-one correspondence between the tangles of order at most 3 and the connected, biconnected, and triconnected components of a graph. Robertson and Seymour 21] established a one-to-one correspondence between tangles of order 2 and biconnected component. Here, we extend the picture to tangles of order 31

\footnotetext{
${ }^{1} \mathrm{My}$ guess is that the result for tangles of order 3 is known to other researchers in the field, but I am not aware of it being published anywhere.
} 


\subsection{Biconnected and Triconnected Components}

Let $G$ be a graph. Following [2, we call a set $X \subseteq V(G) k$-inseparable in $G$ if $|X|>k$ and there is no separation $(A, B)$ of $G$ of order at most $k$ such that $X \backslash V(B) \neq \emptyset$ and $X \backslash V(A) \neq \emptyset$. A $k$-block of $G$ is an inclusionwise maximal $k$-inseparable subset of $V(G)$. We call a $k$-inseparable set of cardinality greater than $k+1$ a proper $k$-inseparable set and, if it is a $k$-block, a proper $k$-block. (Recall that a $(k+1)$-connected graph has order greater than $k+1$ by definition.) We observe that every vertex $x$ in a proper $k$-inseparable set $X$ has degree at least $(k+1)$, because it has $(k+1)$ internally disjoint paths to $X \backslash\{x\}$.

A biconnected component of $G$ is a subgraph induced by a 1-block, which is usually just called a block 2 It is easy to see that a biconnected component $B$ either consists of a single edge that is a bridge of $G$, or it is 2 -connected. In the latter case, we call $B$ a proper biconnected component.

The definition of triconnected components is more complicated, because the subgraph induced by a 2-block is not necessarily 3-connected (even if it is a proper 2-block).

Example 4.1. Let $G$ be a graph obtained from the complete graph $K_{4}$ by subdividing each edge once. Then the vertices of the original $K_{4}$, which are precisely the vertices of degree 3 in $G$, form a proper 2-block, but the subgraph they induce has no edges and thus is certainly not 3 -connected.

It can be shown, however, that every proper 2-block of $G$ is the vertex set of a 3connected topological subgraph. For a subset $X \subseteq V(G)$, we define the torso of $X$ in $G$ to be the graph $G \llbracket X \rrbracket$ obtained from the induced subgraph $G[X]$ by adding an edge $v w$ for all distinct $v, w \in X$ such that there is a connected component $C$ of $G \backslash X$ with $v, w \in N(C)$. We call the edges in $E(G \llbracket X \rrbracket) \backslash E(G)$ the virtual edges of $G \llbracket X \rrbracket$. It is not hard to show that if $X$ is a 2-block of $G$ then for every connected component $C$ of $G \backslash X$ it holds that $N(C) \leq 2$; otherwise $X$ would not be an inclusionwise maximal 2 -inseparable set. This implies that $G \llbracket X \rrbracket$ is a topological subgraph of $G$ : if, for some connected component $C$ of $G \backslash X, N(C)=\{v, w\}$ and hence $v w$ is a virtual edge of the torso, then there is a path from $v$ to $w$ in $C$, which may be viewed as a subdivision of the edge $v w$ of $G \llbracket X \rrbracket$. We call the torsos $G \llbracket X \rrbracket$ for the 2-blocks $X$ the triconnected components of $G$. We call a triconnected component proper if its order is at least 4 .

It is a well known fact, going back to MacLane [15] and Tutte [25], that all graphs admit tree decompositions into their biconnected and triconnected components. Hopcroft and Tarjan [24, 12] proved that the decompositions can be computed in linear time.

\subsection{From Components to Tangles}

Lemma 4.2. Let $G$ be a graph and $X \subseteq V(G)$ a $(k-1)$-inseparable set of order $|X|>$ $\frac{3}{2} \cdot(k-1)$. Then

$$
\mathcal{T}^{(k)}(X):=\{(A, B) \mid(A, B) \text { separation of } G \text { of order }<k \text { with } X \subseteq V(B)\}
$$

is a $G$-tangle of order $k$.

Proof. $\mathcal{T}^{(k)}(X)$ trivially satisfies (GT.0), It satisfies (GT.1) because the $(k-1)$ inseparability of $X$ implies that for every separation $(A, B)$ of $G$ of order $<k$ either $X \subseteq V(A)$ or $X \subseteq V(B)$.

To see that $\overline{\mathcal{T}}^{(k)}(X)$ satisfies $(\mathrm{GT} .2)$, let $\left(A_{i}, B_{i}\right) \in \mathcal{T}^{k}(X)$ for $i=1,2,3$. Then $\left|V\left(A_{i}\right) \cap X\right| \leq k-1$, because $V\left(A_{i}\right) \cap X \subseteq V\left(A_{i}\right) \cap V\left(B_{i}\right)$. As $|X|>\frac{3}{2} \cdot(k-1)$,

\footnotetext{
${ }^{2}$ There is a slight discrepancy to standard terminology here: a set consisting of a single isolated vertex is usually also called a block, but it is not a 1-block, because its size is not greater than 1 .
} 
there is a vertex $x \in X$ such that $x$ is contained in at most one of the sets $V\left(A_{i}\right)$. Say, $x \notin V\left(A_{2}\right) \cup V\left(A_{3}\right)$. If $x \notin V\left(A_{1}\right)$, then $V\left(A_{1}\right) \cup V\left(A_{2}\right) \cup V\left(A_{3}\right) \neq V(G)$. So let us assume that $x \in V\left(A_{1}\right)$.

Let $y_{1}, \ldots, y_{k-1} \in X \backslash\{x\}$. As $X$ is $(k-1)$-inseparable, for all $i$ there is a path $P_{i}$ from $x$ to $y_{i}$ such that $V\left(P_{i}\right) \cap V\left(P_{j}\right)=\{x\}$ for $i \neq j$. Let $w_{i}$ be the last vertex of $P_{i}$ (in the direction from $x$ to $y_{i}$ ) that is in $V\left(A_{1}\right)$. We claim that $w_{i} \in V\left(B_{1}\right)$. This is the case if $w_{i}=y_{i} \in X \subseteq V\left(B_{1}\right)$. If $w_{i} \neq y_{i}$, let $z_{i}$ be the successor of $w_{i}$ on $P_{i}$. Then $z_{i} \in V\left(B_{1}\right) \backslash V\left(A_{1}\right)$, and as $w_{i} z_{i} \in E(G)$, it follows that $w_{i} \in V\left(B_{1}\right)$ as well.

Thus $\left\{x, w_{1}, \ldots, w_{k-1}\right\} \subseteq V\left(A_{1}\right) \cap V\left(B_{1}\right)$, and as $\left|V\left(A_{1}\right) \cap V\left(B_{1}\right)\right| \leq k-1$, it follows that $w_{i}=x$ for some $i$. Consider the edge $e=x z_{i}$. We have $e \notin E\left(A_{1}\right)$ because $z_{i} \notin V\left(A_{1}\right)$ and $e \notin E\left(A_{2}\right) \cup E\left(A_{3}\right)$ because $x \notin V\left(A_{2}\right) \cup V\left(A_{3}\right)$. Hence $E\left(A_{1}\right) \cup E\left(A_{2}\right) \cup E\left(A_{3}\right) \neq E(G)$, and this completes the proof of (GT.2)

Finally, $\mathcal{T}^{(k)}(X)$ satisfies (GT.3) because for every $(A, B) \in \mathcal{T}$ we have $|V(A) \cap X| \leq$ $k-1<|X|$.

Corollary 4.3. Let $G$ be a graph and $X \subseteq V(G)$.

(1) If $X$ is the vertex set of a connected component of $G$ (that is, a 0-block), then $\mathcal{T}^{1}(X)$ is a $G$-tangle of order 1.

(2) If $X$ is the vertex set of a biconnected component of $G$ (that is, a 1-block), then $\mathcal{T}^{2}(X)$ is a $G$-tangle of order 2 .

(3) If $X$ is the vertex set of a proper triconnected component of $G$ (that is, a 2-block of cardinality at least 4$)$, then $\mathcal{T}^{3}(X)$ is a $G$-tangle of order 3 .

Let us close this section by observing that the restriction to proper triconnected components in assertion (3) of the corollary is necessary.

Lemma 4.4. Let $G$ be a graph and $X \subseteq V(G)$ be a 2-block of cardinality 3 . Then $\mathcal{T}^{3}(X)$ is not a tangle.

Proof. Let $\mathcal{T}:=\mathcal{T}^{3}(X)$. Suppose that $X=\left\{x_{1}, x_{2}, x_{3}\right\}$. For $i \neq j$, let $S_{i j}:=\left\{x_{i}, x_{j}\right\}$, and let $Y_{i j}$ be the union of the vertex sets of all connected components $C$ of $G \backslash X$ with $N(C) \subseteq S_{i j}$, and let $Z_{i j}:=V(G) \backslash\left(Y_{i j} \cup S_{i j}\right)$. Let $A_{i j}:=G\left[Y_{i j} \cup S_{i j}\right]$, and let $B_{i j}$ be the graph with vertex set $S_{i j} \cup Z_{i j}$ and edge set $E(G) \backslash E\left(A_{i j}\right)$. Then $\left(A_{i j}, B_{i j}\right) \in \mathcal{T}$, because $X \subseteq V\left(B_{i j}\right)$. As $X$ is a 2-block, for every connected component $C$ of $G \backslash X$ it holds that $|N(C)| \leq 2$, and hence $C \subseteq A_{i j}$ for some $i, j$. It is not hard to see that this implies $A_{12} \cup A_{13} \cup A_{23}=G$. Thus $\mathcal{T}$ violates (GT.2).

\subsection{From Tangles to Components}

For a $G$-tangle $\mathcal{T}$, we let

$$
X_{\mathcal{T}}:=\bigcap_{(A, B) \in \mathcal{T}} V(B) .
$$

In general, $X_{\mathcal{T}}$ may be empty; an example is the tangle of order $k$ associated with a $(k \times k)$-grid for $k \geq 5$ (see Example 3.3). However, it turns out that for tangles of order $k \leq 3$, the set $X_{\mathcal{T}}$ is a $(k-1)$-block. This will be the main result of this section.

Lemma 4.5. Let $\mathcal{T}$ be a $G$-tangle of order $k$. If $\left|X_{\mathcal{T}}\right| \geq k$, then $X_{\mathcal{T}}$ is a $(k-1)$-block of $G$ and $\mathcal{T}=\mathcal{T}^{k}\left(X_{\mathcal{T}}\right)$.

Proof. Suppose that $\left|X_{\mathcal{T}}\right| \geq k$. If $(A, B)$ is a separation of $G$ of order less than $k$ then either $(A, B) \in \mathcal{T}$ or $(B, A) \in \mathcal{T}$, which implies $X_{\mathcal{T}} \subseteq V(B)$ or $X_{\mathcal{T}} \subseteq V(A)$. Thus $X_{\mathcal{T}}$ is $(k-1)$-inseparable. If $X \supset X_{\mathcal{T}}$, say, with $x \in X \backslash X_{\mathcal{T}}$, then there is some separation 


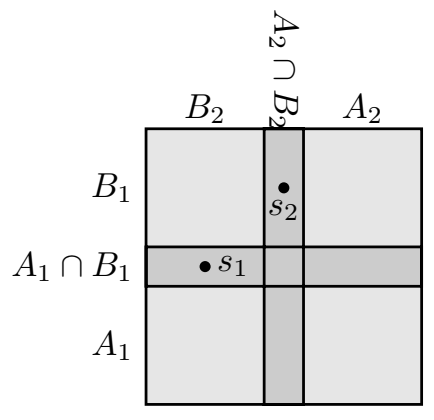

Figure 4.1. Proof of Theorem 4.6

$(A, B) \in \mathcal{T}$ with $x \in V(A) \backslash V(B)$ and $X_{\mathcal{T}} \subseteq V(B)$, and this implies that $X$ is not $(k-1)$-inseparable. Hence $X_{\mathcal{T}}$ is a $k$-block.

We have $\mathcal{T}=\mathcal{T}^{k}\left(X_{\mathcal{T}}\right)$, because $X_{\mathcal{T}} \subseteq V(B)$ for all $(A, B) \in \mathcal{T}$, and for a separation $(A, B)$ of order at most $k-1$ we cannot have $X_{\mathcal{T}} \subseteq V(A) \cap V(B)$.

Let $\mathcal{T}$ be a $G$-tangle. A separation $(A, B) \in \mathcal{T}$ is minimal in $\mathcal{T}$ if there is no $\left(A^{\prime}, B^{\prime}\right) \in \mathcal{T}$ such that $B^{\prime} \subset B$. Clearly, $X_{\mathcal{T}}$ is the intersection of all sets $V(B)$ for minimal $(A, B) \in \mathcal{T}$. Hence if we want to understand $X_{\mathcal{T}}$, we can restrict our attention to the minimal separations in $\mathcal{T}$. Let $(A, B) \in \mathcal{T}$ be minimal and $S:=V(A) \cap V(B)$. It follows from Lemma 3.6 that $B \backslash S=C:=C(\mathcal{T}, S)$, and it follows from the minimality that $S=N(C)$ and that $E(B)$ consists of all edges with one endvertex in $V(C)$. Hence $B$ is connected.

Theorem 4.6 (Robertson and Seymour [21]). Let $G$ be a graph.

(1) For every $G$-tangle $\mathcal{T}$ of order 1 , the set $X_{\mathcal{T}}$ is a vertex set of a connected component of $G$, and we have $\mathcal{T}=\mathcal{T}^{1}\left(X_{\mathcal{T}}\right)$.

(2) For every $G$-tangle $\mathcal{T}$ of order 2 , the set $X_{\mathcal{T}}$ is the vertex set of a biconnected component of $G$, and we have $\mathcal{T}=\mathcal{T}^{2}\left(X_{\mathcal{T}}\right)$.

Proof. To prove (1), let $\mathcal{T}$ be a $G$-tangle of order 1 . Let $C=C(\mathcal{T}, \emptyset)$. Then $(G \backslash V(C), C)$ is the unique minimal separation in $\mathcal{T}$, and thus we have $X_{\mathcal{T}}=V(C)$.

To prove (2), let $\mathcal{T}$ be a $G$-tangle of order 2. By Lemma 4.5, it suffices to prove that $\left|X_{\mathcal{T}}\right| \geq 2$. Let $\mathcal{T}^{\prime}$ be the truncation of $\mathcal{T}$ to order 1 . Then $W:=X_{\mathcal{T}}$, is the vertex set of a connected component $C$ of $G$, and we have $X_{\mathcal{T}} \subseteq W$. Moreover, for every minimal $(A, B) \in \mathcal{T}$ we have $B \subseteq C$, because $B$ is connected and $B \cap C \neq \emptyset$ by (GT.2).

Claim 1. Let $\left(A_{1}, B_{1}\right),\left(A_{2}, B_{2}\right) \in \mathcal{T}$ be distinct and minimal in $\mathcal{T}$. Then $A_{1} \cap C \subseteq B_{2}$ and $A_{2} \cap C \subseteq B_{1}$.

Proof. We have ord $\left(A_{1} \cup A_{2}, B_{1} \cap B_{2}\right) \geq 2$, because otherwise $\left(A_{1} \cup A_{2}, B_{1} \cap B_{2}\right) \in \mathcal{T}$ by Lemma 3.4 (3), which contradicts the minimality of the separations $\left(A_{i}, B_{i}\right)$. Suppose that $V\left(A_{i}\right) \cap V\left(B_{i}\right)=\left\{s_{i}\right\}$. As

$$
V\left(A_{1} \cup A_{2}\right) \cap\left(V\left(B_{1} \cap B_{2}\right) \subseteq V\left(A_{1} \cap B_{1}\right) \cup V\left(A_{2} \cap B_{2}\right)=\left\{s_{1}, s_{2}\right\},\right.
$$

we must have $s_{1} \neq s_{2}$ and $V\left(A_{1} \cup A_{2}\right) \cap V\left(B_{1} \cap B_{2}\right)=\left\{s_{1}, s_{2}\right\}$ (see Figure 4.1). This implies $V\left(A_{1} \cap A_{2}\right) \cap V\left(B_{1} \cup B_{2}\right)=\emptyset$. Then $\left(A_{1} \cap A_{2}, B_{1} \cup B_{2}\right)$ is a separation of $G$ of order 0 , and as $C$ is connected and $\left(B_{1} \cup B_{2}\right) \cap C \neq \emptyset$, we have $A_{1} \cap A_{2} \cap C=\emptyset$. The assertion of the claim follows. 
Let $\left(A_{1}, B_{1}\right), \ldots,\left(A_{m}, B_{m}\right)$ be an enumeration of all minimal separations in $\mathcal{T}$ of order 1. Even if $C$ is 1-inseparable, there is such a separation: $(G \backslash(V(C) \backslash\{v\}), C)$ for an arbitrary $v \in V(C)$. Thus $m \geq 1$. If $m=1$, then $X_{\mathcal{T}}=V\left(B_{1}\right)$ and thus $\left|X_{\mathcal{T}}\right| \geq 2$ by Lemma 3.4(1).

If $m \geq 2$, let $A_{i} \cap B_{i}=\left\{s_{i}\right\}$. We can assume the $s_{i}$ to be mutually distinct, because if $s_{i}=s_{j}$ then $B_{i}=B_{j}$. It follows from Claim 1 that $s_{1}, \ldots, s_{m} \in \bigcap_{i} V\left(B_{i}\right)=X_{\mathcal{T}}$. This implies $\left|X_{\mathcal{T}}\right| \geq 2$.

To extend Theorem 4.6 to tangles of order 3 , we first prove a lemma, which essentially says that we can restrict our attention to 2-connected graphs. Let $G$ be graph and $X \subseteq$ $V(G)$. For every $A \subseteq G$, let $A \cap X:=A[V(A) \cap X]$. Note that if $(A, B)$ is a separation of $G$, then $(A \cap X, B \cap X)$ is a separation of $G[X]$ with $\operatorname{ord}(A \cap X, B \cap X) \leq \operatorname{ord}(A, B)$.

Lemma 4.7. Let $\mathcal{T}$ be a $G$-tangle of order 3 . Let $\mathcal{T}^{\prime}$ be the truncation of $\mathcal{T}$ to order 2, and let $W:=X_{\mathcal{T}^{\prime}}$. Let $\mathcal{T}[W]$ be the set of all separations $(A \cap W, B \cap W)$ of $G[W]$ where $(A, B) \in \mathcal{T}$. Then $\mathcal{T}[W]$ is a $G[W]$-tangle of order 3 . Furthermore, $X_{\mathcal{T}}=X_{\mathcal{T}[W]}$.

Proof. By Theorem [4.6, $G[W]$ is a biconnected component of $G$. This implies that $|W| \geq 2$ and $|N(C)| \leq 1$ for every connected component $C$ of $G \backslash W$. For every $w \in W$, we let $Y_{w}$ be union of the vertex sets of all connected components $C$ of $G \backslash W$ with $N(C) \subseteq\{w\}$. Then $V(G)=W \cup \bigcup_{w \in W} Y_{w}$. Let $Z_{w}:=V(G) \backslash\left(Y_{w} \cup\{w\}\right)$. Let $A_{w}:=G\left[Y_{w} \cup\{w\}\right]$ and $B_{w}:=G\left[Z_{w} \cup\{w\}\right]$. Then $W \subseteq V\left(B_{w}\right)$ and thus $\left(A_{w}, B_{w}\right) \in$ $\mathcal{T}^{2}(W)=\mathcal{T}^{\prime} \subseteq \mathcal{T}$.

Claim 1. Let $(A, B) \in \mathcal{T}$. Then $W \backslash V(A) \neq \emptyset$.

Proof. Suppose for contradiction that $W \subseteq V(A)$. Let $S:=V(A) \cap V(B)$ and suppose that $S=\left\{s_{1}, s_{2}\right\}$. Let $w_{i} \in W$ such that $s_{i} \in Y_{w_{i}} \cup\left\{w_{i}\right\}$. Then $A \cup A_{w_{1}} \cup A_{w_{2}}=G$, which contradicts (GT.2). This proves that $W \backslash V(A) \neq \emptyset$.

It is now straightforward to prove that $\mathcal{T}[W]$ satisfies the tangle axioms (GT.0) (GT.1), and (GT.3) To prove (GT.2), let $\left(A_{i}, B_{i}\right) \in \mathcal{T}$ for $i=1,2,3$. We need to prove that $\left(A_{1} \cap W\right) \cup\left(A_{2} \cap W\right) \cup\left(A_{3} \cap W\right) \neq G[W]$. Without loss of generality we may assume that $\left(A_{i}, B_{i}\right)$ is minimal in $\mathcal{T}$. Then $C_{i}:=B_{i} \backslash V\left(A_{i}\right)$ is connected. By Claim 1, $V\left(C_{i}\right) \cap W \neq \emptyset$. This implies that if $V\left(C_{i}\right) \cap Y_{w} \neq \emptyset$ for some $w \in W$, then $w \in V\left(C_{i}\right)$.

As $\mathcal{T}$ satisfies (GT.2), $A_{1} \cup A_{2} \cup A_{3} \neq G$, and thus there either is a vertex in $V\left(C_{1}\right) \cap V\left(C_{2}\right) \cap V\left(C_{3}\right)$ or an edge with an endvertex in every $V\left(C_{i}\right)$. Suppose first that $v \in V\left(C_{1}\right) \cap V\left(C_{2}\right) \cap V\left(C_{3}\right)$. If $v \in W$ then

$$
V\left(\left(A_{1} \cap W\right) \cup\left(A_{2} \cap W\right) \cup\left(A_{3} \cap W\right)\right) \neq W=V(G[W]) .
$$

Otherwise, $v \in Y_{w}$ for some $w \in W$, and we have $w \in V\left(C_{1}\right) \cap V\left(C_{2}\right) \cap V\left(C_{3}\right)$. Similarly, if $e=v v^{\prime}$ has an endvertex in every $V\left(C_{i}\right)$, then we distinguish between the case that $v, v^{\prime} \in W$, which implies $E\left(\left(A_{1} \cap W\right) \cup\left(A_{2} \cap W\right) \cup\left(A_{3} \cap W\right)\right) \neq E(G[W])$, and the case that $e \in E\left(A_{w}\right)$ for some $w \in W$, which implies $w \in V\left(C_{1}\right) \cap V\left(C_{2}\right) \cap V\left(C_{3}\right)$ and thus $V\left(\left(A_{1} \cap W\right) \cup\left(A_{2} \cap W\right) \cup\left(A_{3} \cap W\right)\right) \neq W=V(G[W])$. This proves (GT.2) and hence that $\mathcal{T}[W]$ is a tangle.

The second assertion $X_{\mathcal{T}}=X_{\mathcal{T}[W]}$ follows from the fact that $X_{\mathcal{T}} \subseteq X_{\mathcal{T}^{\prime}}=W$.

Theorem 4.8. Let $G$ be a graph. For every $G$-tangle $\mathcal{T}$ of order 3 , the set $X_{\mathcal{T}}$ is a vertex set of a proper triconnected component of $G$.

Proof. Let $\mathcal{T}$ be a $G$-tangle of order 3. It suffices to prove that $\left|X_{\mathcal{T}}\right| \geq 3$. Then by Lemma 4.5, $X_{\mathcal{T}}$ is a 3 -block and $\mathcal{T}=\mathcal{T}^{3}\left(X_{\mathcal{T}}\right)$, and by Lemma 4.4 $X_{\mathcal{T}}$ is proper 3-block, that is, the vertex set of a proper triconnected component. 


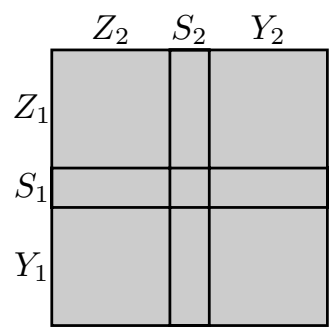

(a)

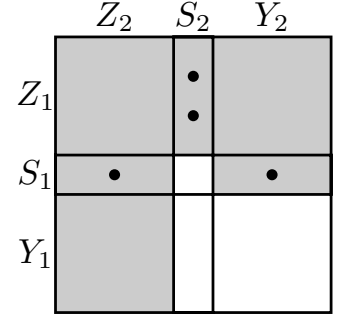

(b)

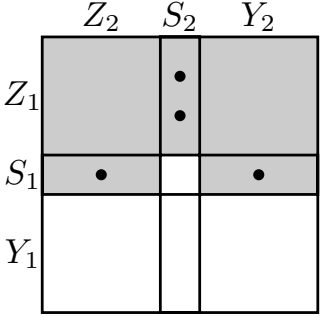

(c)

Figure 4.2. Uncrossing minimal separations of order 2

By the previous lemma, we may assume without loss of generality that $G$ is 2connected. The rest of the proof follows the lines of the proof of Theorem 4.6 The core of the proof is again an "uncrossing argument" (this time a more complicated one) in Claim 1.

Claim 1. Let $\left(A_{1}, B_{1}\right),\left(A_{2}, B_{2}\right) \in \mathcal{T}$ be distinct and minimal in $\mathcal{T}$. Then $V\left(A_{1}\right) \subseteq$ $V\left(B_{2}\right)$ and $V\left(A_{2}\right) \subseteq V\left(B_{1}\right)$.

Proof. Let $S_{i}:=V\left(A_{i}\right) \cap V\left(B_{i}\right)$ and $Y_{i}:=V\left(A_{i}\right) \backslash S_{i}$ and $Z_{i}:=V\left(B_{i}\right) \backslash S_{i}$ (see Figure 4.2(a)). By the minimality of $\left(A_{i}, B_{i}\right)$, we have $Z_{i}=V\left(C\left(\mathcal{T}, S_{i}\right)\right)$ and $S_{i}=$ $N\left(Z_{i}\right)$. Thus $S_{1} \neq S_{2}$ and $Z_{1} \neq Z_{2}$, because the two separations are distinct.

It follows that $\left(A_{1} \cup A_{2}, B_{1} \cap B_{2}\right)$ is a separation with $B_{1} \cap B_{2} \subset B_{i}$, and by the minimality of $\left(A_{i}, B_{i}\right)$ this separation is not in $\mathcal{T}$. By Lemma 3.4(3), this means that its order is at least 3 . Thus

$$
\left|S_{1} \cap Z_{2}\right|+\left|S_{1} \cap S_{2}\right|+\left|Z_{1} \cap S_{2}\right|=\left|V\left(A_{1} \cup A_{2}\right) \cap V\left(B_{1} \cap B_{2}\right)\right| \geq 3 .
$$

As $\left|S_{i}\right| \leq 2$ and $S_{1} \neq S_{2}$, it follows that

$$
\left|S_{1} \cap Y_{2}\right|+\left|S_{1} \cap S_{2}\right|+\left|Y_{1} \cap S_{2}\right|=\left|V\left(A_{1} \cap A_{2}\right) \cap V\left(B_{1} \cup B_{2}\right)\right| \leq 1 .
$$

Hence $\left(A_{1} \cap A_{2}, B_{1} \cup B_{2}\right)$ is a separation of order at most 1 . As $G$ is 2-connected, the separation is not proper, which means that either $V\left(A_{1} \cap A_{2}\right)=V(G)$ or $V\left(B_{1} \cup B_{2}\right)=$ $V(G)$. By Lemma 3.4 (2), we have $\left(A_{1} \cap A_{2}, B_{1} \cup B_{2}\right) \in \mathcal{T}$ and thus $V\left(A_{1} \cap A_{2}\right) \neq V(G)$. Thus $V\left(B_{1} \cup B_{2}\right)=V(G)$, and this implies $Y_{1} \cap Y_{2}=\emptyset$.

To prove that $V\left(A_{i}\right)=S_{i} \cup Y_{i} \subseteq V\left(B_{3-i}\right)=S_{3-i} \cup Z_{3-i}$, we still need to prove that $S_{i} \cap Y_{3-i}=\emptyset$. Suppose for contradiction that $S_{1} \cap Y_{2} \neq \emptyset$. Then (因) implies $\left|S_{1} \cap Y_{2}\right|=1$ and $\left|S_{1} \cap Z_{2}\right|=1$ and $\left|S_{2} \cap Z_{1}\right|=2$ and $S_{1} \cap S_{2}=Y_{1} \cap S_{2}=\emptyset$ (see Figure 4.2(b)). Note that $\left(Y_{1} \cup S_{1}\right) \cap Z_{2}=V\left(A_{1}\right) \backslash V\left(A_{2}\right)$. It follows that $\left(A_{1} \backslash V\left(A_{2}\right), B_{1}\right)$ is a separation of $G$ of order 1 , and we have $\left(A_{1} \backslash V\left(A_{2}\right), B_{1}\right) \in \mathcal{T}$. Thus $Y_{1} \cap Z_{2}=\emptyset$, which implies $V\left(B_{2}\right)=Z_{2} \cup S_{2} \subset Z_{1} \cup S_{1}=V\left(B_{1}\right)$ (see Figure 4.2(c)). This contradicts the minimality of $\left(A_{1}, B_{1}\right)$. Hence $S_{1} \cap Y_{2}=\emptyset$, and similarly $Y_{1} \cap S_{2}=\emptyset$.

Let $\left(A_{1}, B_{1}\right), \ldots,\left(A_{m}, B_{m}\right)$ be an enumeration of all minimal separations in $\mathcal{T}$ of order 2. Note that there is at least one minimal separation of order 2 even if $G$ has no proper separations of order 2 . Thus $m \geq 1$.

Let $S_{i}:=V\left(A_{i}\right) \cap V\left(B_{i}\right)$. Then the sets $S_{i}$ are all distinct, because two minimal separations in $\mathcal{T}$ with the same separators are equal. It follows from Claim 1 that $S_{i} \subseteq V\left(B_{j}\right)$ for all $j \in[m]$ and thus

$$
S_{1} \cup \ldots \cup S_{m} \subseteq X_{\mathcal{T}}
$$


If $m \geq 2$ this implies $\left|X_{\mathcal{T}}\right| \geq 3$. If $m=1$, then $X_{\mathcal{T}}=V\left(B_{1}\right)$ and thus $\left|X_{\mathcal{T}}\right| \geq 3$ by Lemma 3.4

The results of this section clearly do not extend beyond tangles of order 3 . For example, the hexagonal grid $H$ in Figure 1.1 has a (unique) tangle $\mathcal{T}$ of order 4 . But the set $X_{\mathcal{T}}$ is empty, and the graph $H$ has no 3 -inseparable set of cardinality greater than 1.

Nevertheless, it is shown in [6] that there is an extension of the theorem to tangles of order 4 if we replace 4-connectivity by the slightly weaker "quasi-4-connectivity": a graph $G$ is quasi-4-connected if it is 3-connected and for all separations $(A, B)$ of order 3, either $|V(A) \backslash V(B)| \leq 1$ or $|V(B) \backslash V(A)| \leq 1$. For example, the hexagonal grid $H$ in Figure 1.1 is quasi-4-connected. It turns out that there is a one-to-one correspondence between the tangles of order 4 and (suitably defined) quasi-4-connected components of a graph.

\section{A Broader Perspective: Tangles and Connectivity Systems}

Many aspects of "connectivity" are not specific to connectivity in graphs, but can be seen in an abstract and much more general context. We describe "connectivity" on some structure as a function that assigns an "order" (a nonnegative integer) to every "separation" of the structure. We study symmetric connectivity functions, where the separations $(A, B)$ and $(B, A)$ have the same order. The key property such connectivity functions need to satisfy is submodularity.

Separations can usually be described as partitions of a suitable set, the "universe". For example, the separations of graphs we considered in the previous sections are essentially partitions of the edge set. Technically, it will be convenient to identify a partition $(\bar{X}, X)$ with the set $X$, implicitly assuming that $\bar{X}$ is the complement of $X$. This leads to the following definition.

A connectivity function on a finite set $U$ is a symmetric and submodular function $\kappa: 2^{U} \rightarrow \mathbb{N}$ with $\kappa(\emptyset)=0$. Symmetric means that $\kappa(X)=\kappa(\bar{X})$ for all $X \subseteq U$; here and whenever the ground set $U$ is clear from the context we write $\bar{X}$ to denote $U \backslash X$. Submodular means that $\kappa(X)+\kappa(Y) \geq \kappa(X \cap Y)+\kappa(X \cup Y)$ for all $X, Y \subseteq U$. The pair $(U, \kappa)$ is sometimes called a connectivity system.

The following two examples capture what is known as edge connectivity and vertex connectivity in a graph.

Example 5.1 (Edge connectivity). Let $G$ be a graph. We define the function $\nu_{G}$ : $2^{V(G)} \rightarrow \mathbb{N}$ by letting $\nu_{G}(X)$ be the number of edges between $X$ and $\bar{X}$. Then $\nu_{G}$ is a connectivity function on $V(G)$.

Example 5.2 (Vertex connectivity). Let $G$ be a graph. We define the function $\kappa_{G}: 2^{E(G)} \rightarrow \mathbb{N}$ by letting $\kappa_{G}(X)$ be the number of vertices that are incident with an edge in $X$ and an edge in $\bar{X}$. Then $\kappa_{G}$ is a connectivity function on $E(G)$.

Note that for all separations $(A, B)$ of $G$ we have $\kappa_{G}(E(A))=\kappa_{G}(E(B)) \leq \operatorname{ord}(A, B)$, with equality if $V(A) \cap V(B)$ contains no isolated vertices of $A$ or $B$. For $X \subseteq E(G)$, let us denote the set of endvertices of the edges in $X$ by $V(X)$. Then for all $X \subseteq E(G)$ we have $\kappa_{G}(X)=\operatorname{ord}\left(A_{X}, B_{X}\right)$, where $B_{X}=(V(X), X)$ and $A_{X}=(V(\bar{X}), \bar{X})$. The theory of tangles and decompositions of the connectivity function of $\kappa_{G}$ is essentially the same as the theory of tangles and decompositions of $G$ (partially developed in the previous sections).

Example 5.3. Let $G$ be a graph. For all subsets $X, Y \subseteq V(G)$, we let $M=M_{G}(X, Y)$ be the $X \times Y$-matrix over the 2-element field $\mathbb{F}_{2}$ with entries $M_{x y}=1 \Longleftrightarrow x y \in E(G)$. Now we define a connectivity function $\rho_{G}$ on $V(G)$ by letting $\rho_{G}(X)$, known as the 
cut rank of $X$, be the row rank of the matrix $M_{G}(X, \bar{X})$. This connectivity function was introduced by Oum and Seymour [17] to define the rank width of graphs, which approximates the clique width, but has better algorithmic properties.

Let us also give an example of a connectivity function not related to graphs.

Example 5.4. Let $M$ be a matroid with ground set $E$ and rank function $r$. (The rank of a set $X \subseteq E$ is defined to be the maximum size of an independent set contained in $X$.) The connectivity function of $M$ is the set function $\kappa_{M}: E \rightarrow \mathbb{N}$ defined by $\kappa_{M}(X)=r(X)+r(\bar{X})-r(E)$ (see, for example, [18).

\subsection{Tangles}

Let $\kappa$ be a connectivity function on a set $U$. A $\kappa$-tangle of order $k \geq 0$ is a set $\mathcal{T} \subseteq 2^{U}$ satisfying the following conditions.

(T.0) $\kappa(X)<k$ for all $X \in \mathcal{T}$,

(T.1) For all $X \subseteq U$ with $\kappa(X)<k$, either $X \in \mathcal{T}$ or $\bar{X} \in \mathcal{T}$.

(T.2) $X_{1} \cap X_{2} \cap X_{3} \neq \emptyset$ for all $X_{1}, X_{2}, X_{3} \in \mathcal{T}$.

(T.3) $\mathcal{T}$ does not contain any singletons, that is, $\{a\} \notin \mathcal{T}$ for all $a \in U$.

We denote the order of a $\kappa$-tangle $\mathcal{T}$ by $\operatorname{ord}(\mathcal{T})$.

We mentioned in Example 5.2 that the theory of $\kappa_{G}$-tangles is essentially the same as the theory of tangles in a graph. Indeed, $\kappa_{G}$-tangles and $G$-tangles are "almost" the same. The following proposition makes this precise.

We call an edge of a graph isolated if both of its endvertices have degree 1. We call an edge pendant if it is not isolated and has one endvertex of degree 1.

Proposition 5.5. Let $G$ be a graph and $k \geq 0$.

(1) If $\mathcal{T}$ is a $\kappa_{G}$-tangle of order $k$, then

$$
\mathcal{S}:=\{(A, B) \mid(A, B) \text { separation of } G \text { of order }<k \text { with } E(B) \in \mathcal{T}\}
$$

is a $G$-tangle of order $k$.

(2) If $\mathcal{S}$ is a $G$-tangle of order $k$, then

$$
\mathcal{T}:=\{E(B) \mid(A, B) \in \mathcal{S}\}
$$

is a $\kappa_{G}$-tangle of order $k$, unless

(i) either $k=1$ and there is an isolated vertex $v \in V(G)$ such that $\mathcal{S}$ is the set of all separations $(A, B)$ of order 0 with with $v \in V(B) \backslash V(A)$,

(ii) or $k=1$ and there is an isolated edge $e \in E(G)$ such that $\mathcal{S}$ is the set of all separations $(A, B)$ of order 0 with $e \in E(B)$,

(iii) or $k=2$ and there is an isolated or pendant edge $e=v w \in E(G)$ and $\mathcal{S}$ is the set of all separations $(A, B)$ of order at most 1 with $e \in E(B)$.

We omit the straightforward (albeit tedious) proof.

Example 5.6. Let $G$ be the graph shown in Figure 5.1 $G$ has one tangle of order 1 (since it is connected) and three tangles of order 2 corresponding to the three biconnected components. The $G$-tangle corresponding to the "improper" biconnected component consisting of the edge $e_{1}$ and its endvertices does not correspond to a $\kappa_{G}$-tangle (by Proposition 5.5(2-iii)). 


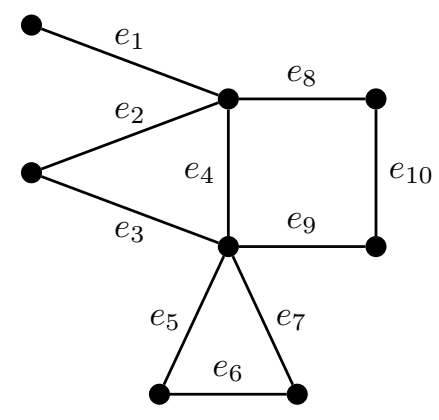

Figure 5.1. A graph $G$ with three $G$ tangles of order 2 and two $\kappa_{G}$-tangles of order 2

A star is a connected graph in which at most 1 vertex has degree greater than 1 . Note that we admit degenerate stars consisting of a single vertex or a single edge.

Corollary 5.7. Let $G$ be a graph that has a $G$-tangle of order $k$. Then $G$ has a $\kappa_{G^{-}}$ tangle of order $k$, unless $k=1$ and $G$ only has isolated edges or $k=2$ and all connected components of $G$ are stars.

\section{Decompositions and Duality}

A cubic tree is a tree where every node that is not a leaf has degree 3 . An oriented edge of a tree $T$ is a pair $(s, t)$, where $s t \in E(T)$. We denote the set of all oriented edges of $T$ by $\vec{E}(T)$ and the set of leaves of $T$ by $L(T)$. A branch decomposition of a connectivity function $\kappa$ over $U$ is a pair $(T, \xi)$, where $T$ is a cubic tree and $\xi$ a bijective mapping from $L(T)$ to $U$. For every oriented edge $(s, t) \in \vec{E}(T)$ we define $\widetilde{\xi}(s, t)$ to be the set of all $\xi(u)$ for leaves $u \in L(T)$ contained in the same connected component of $T-\{s t\}$ as $t$. Note that $\widetilde{\xi}(s, t)=\overline{\widetilde{\xi}(t, s)}$. We define the width of the decomposition $(T, \xi)$ be the maximum of the values $\kappa(\widetilde{\xi}(t, u))$ for $(t, u) \in \vec{E}(T)$. The branch width of $\kappa$, denoted by $\mathrm{bw}(\kappa)$, is the minimum of the widths of all its branch decompositions.

The following fundamental result relates tangles and branch decompositions; it is one of the reasons why tangles are such interesting objects.

Theorem 6.1 (Duality Theorem; Robertson and Seymour [21]). The branch width of a connectivity function $\kappa$ equals the maximum order of a $\kappa$-tangle.

We omit the proof.

Let $G$ be a graph. A branch decomposition of $G$ is defined to be a branch decomposition of $\kappa_{G}$, and the branch width of $G$, denoted by bw $(G)$, is the branch width of $\kappa_{G}$.

Example 6.2. Let $G$ be the graph shown in Figure 5.1, Figure 6.1 shows a branch decomposition of $G$ of width 2. Thus bw $(G) \leq 2$. As $G$ has a tangle of order 2 (see Example (5.6), by the Duality Theorem we have bw $(G)=2$.

The branch width of a graph is closely related to the better-known tree width $\operatorname{tw}(G)$ : it is not difficult to prove that

$$
\mathrm{bw}(G) \leq \mathrm{tw}(G)+1 \leq \max \left\{\frac{3}{2} \mathrm{bw}\left(\kappa_{G}\right), 2\right\}
$$

(Robertson and Seymour [21). Both inequalities are tight. For example, a complete graph $K_{3 n}$ has branch width $2 n$ and tree width $3 n-1$, and a path of length 3 has branch 


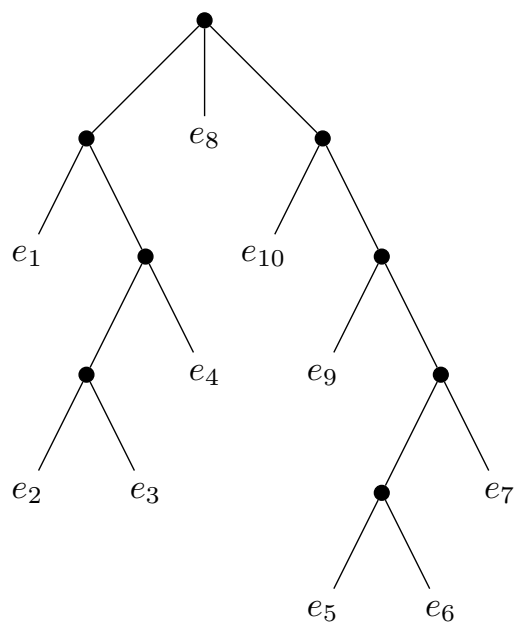

Figure 6.1. A branch decomposition of width 2 of the graph shown in Figure 5.1

width 2 and tree width 1 . There is also a related duality theorem for tree width, due to Seymour and Thomas [23]: $\operatorname{tw}(G)+1$ equals the maximum order of bramble of $G$. (Recall the characterisation of tangles that we gave in Theorem 3.7 and the definition of brambles right after the theorem.)

\section{Acknowledgements}

I thank Pascal Schweitzer and Konstantinos Stavropoulos for helpful comments on a earlier version of the paper.

\section{References}

[1] J. Carmesin, R. Diestel, M. Hamann, and F. Hundertmark. Canonical tree-decompositions of finite graphs I. Existence and algorithms. ArXiv, arXiv:1305.4668v3 [math.CO], 2013.

[2] J. Carmesin, R. Diestel, F. Hundertmark, and M. Stein. Connectivity and tree structure in finite graphs. Combinatorica, 34(1):11-46, 2014.

[3] E.D. Demaine, M.T. Hajiaghayi, and K. Kawarabayashi. Algorithmic graph minor theory: Decomposition, approximation, and coloring. In Proceedings of the 45 th Annual IEEE Symposium on Foundations of Computer Science, pages 637-646, 2005 .

[4] R. Diestel. Graph Theory. Springer, 4th edition, 2010.

[5] J. Geelen, B. Gerards, and G. Whittle. Tangles, tree-decompositions and grids in matroids. Journal of Combinatorial Theory, Series B, 99(4):657-667, 2009.

[6] M. Grohe. Quasi-4-connected components. ArXiv, 2016.

[7] M. Grohe, K. Kawarabayashi, and B. Reed. A simple algorithm for the graph minor decomposition - logic meets structural graph theory. In Proceedings of the 24th Annual ACM-SIAM Symposium on Discrete Algorithms, pages 414-431, 2013. 
[8] M. Grohe and D. Marx. Structure theorem and isomorphism test for graphs with excluded topological subgraphs. In Proceedings of the 44th ACM Symposium on Theory of Computing, 2012.

[9] M. Grohe and D. Marx. Structure theorem and isomorphism test for graphs with excluded topological subgraphs. SIAM Journal on Computing, 44(1):114-159, 2015.

[10] M. Grohe and P. Schweitzer. Computing with tangles. In Proceedings of the 47th ACM Symposium on Theory of Computing, pages 683-692, 2015.

[11] M. Grohe and P. Schweitzer. Isomorphism testing for graphs of bounded rank width. In Proceedings of the 55th Annual IEEE Symposium on Foundations of Computer Science, 2015.

[12] J. E. Hopcroft and R. Tarjan. Dividing a graph into triconnected components. SIAM Journal on Computing, 2(2):135-158, 1973.

[13] F. Hundertmark. Profiles. An algebraic approach to combinatorial connectivity. ArXiv, arXiv:1110.6207v1 [math.CO], 2011.

[14] K.-I. Kawarabayashi and P. Wollan. A simpler algorithm and shorter proof for the graph minor decomposition. In Proceedings of the 43rd ACM Symposium on Theory of Computing, pages 451-458, 2011.

[15] S. MacLane. A structural characterization of planar combinatorial graphs. Duke Mathematical Journal, 3(3):460-472, 1937.

[16] S.-I. Oum. Rank-width and vertex-minors. Journal of Combinatorial Theory, Series $B, 95: 79-100,2005$.

[17] S.-I. Oum and P.D. Seymour. Approximating clique-width and branch-width. Journal of Combinatorial Theory, Series B, 96:514-528, 2006.

[18] J. Oxley. Matroid Theory. Cambridge University Press, 2nd edition, 2011.

[19] B. Reed. Tree width and tangles: A new connectivity measure and some applications. In R.A. Bailey, editor, Surveys in Combinatorics, volume 241 of LMS Lecture Note Series, pages 87-162. Cambridge University Press, 1997.

[20] N. Robertson and P.D. Seymour. Graph minors I-XXIII. Journal of Combinatorial Theory, Series B 1982-2012.

[21] N. Robertson and P.D. Seymour. Graph minors X. Obstructions to treedecomposition. Journal of Combinatorial Theory, Series B, 52:153-190, 1991.

[22] N. Robertson and P.D. Seymour. Graph minors XVI. Excluding a non-planar graph. Journal of Combinatorial Theory, Series B, 77:1-27, 1999.

[23] P.D. Seymour and R. Thomas. Graph searching and a min-max theorem for treewidth. Journal of Combinatorial Theory, Series B, 58:22-33, 1993.

[24] R. Tarjan. Depth-first search and linear graph algorithms. SIAM Journal on Computing, 1(2):146-160, 1972.

[25] W.T. Tutte. Graph Theory. Addison-Wesley, 1984. 